\title{
Analysis of QOS Parameters of AAMRP and IODMRP using SBPGP Security Model
}

\author{
Ranjeet Singh \\ Computer Science Engineering \\ Lovely Professional University, \\ Phagwara, India
}

\author{
Harwant Singh Arri \\ Computer Science Engineering \\ Lovely Professional University, \\ Phagwara, India
}

\begin{abstract}
MANET has gained popularity in various fields as in vehicles, data transmission and bulk data transmission to a large single set of nodes. To accomplish this task routing protocol employ an important role in MANET. Different types of routing protocols have been proposed for different task to be done. Most of the parameters have been considered during the establishment of routing protocols. It is concerned with the various factors such as energy efficiency, routing protocols, security and various other factors. In the current work we are going to work with multicast routing protocol (IODMR and AAMRP) with security as major aspect. Multicasting is the ability to send packets to and receive packets directed at a subset of nodes in a network. We are applying the SBPGP model with these protocol and will find out their performance under certain conditions with various malicious attacks can be handled efficiently on MANET by using different size of encryption bit keys on the basis of throughput, end-to-end delay and packet delivery fraction.
\end{abstract}

\section{Keywords}

\section{IODMR, AAMRP, SBPGP, MANET}

\section{INTRODUCTION}

The main objectives of MANET routing protocols are to maximize network throughput, to maximize network lifetime, to maximize energy efficiency and to minimize delays. The network throughput is measured by packet delivery ratio and energy contribution is measured by routing overhead which is number or size of routing control packets.

Need of security for routing protocols:

Mobile ad-hoc networks are vulnerable to attacks that differ from those in fixed networks their properties pose additional requirements to security and cooperation protocols.

[2] Introduces and classifies several proposals in the field of constructing multicast concept in ad hoc wireless networks (MANET's). The classification of these protocols is performed according to several points of view as topology structure, core versus coreless approaches, initialization of multicast session, dependency on unicast routing protocols and topology maintenance approaches.

Multicasting is the ability to send packets to and receive packets directed at a subset of nodes in a network. [7]In general multicast routing is achieved using either:

A. Source based- When no. of multicast senders in a group are small( e.g.-video on demand application)

B. Core based trees- In core based concept a group of members share a multicast tree and Multicast forwarding is based on nodes rather than on links.

\section{MULTICAST ROUTING PROTOCOL UNDER HOOD:}

\subsection{Ant Based Adaptive Multicast Routing Protocol (AAMRP)}

[5] AAMRP dynamically identifies and organizes the group members into clusters which correspond to areas of high group member affinity. In each of these "dense" neighborhoods, one of the group members is selected to be cluster leader.

In [5] Ant agent based adaptive multicast protocol is proposed which combines the positive aspects both broadcasting and multicasting. It exploits group members desire to simplify multicast routing and invoke broadcast operations in appropriate localized regimes. It achieves packet delivery statistics that are comparable to that with a pure multicast protocol but with lower overheads.

Cluster leader have two main functions:

1. They establish a sparse multicast structure among themselves and the source, and

2. They use broadcasting (with adaptive scope) to deliver the packets to other group members in their cluster.

It constructs a 2-tier hierarchical structure:

- Upper tier is formed by a multicast source and cluster leaders that represent groups of multicast members that form a cluster.

- Lower tier consists of the members in a cluster.

Each cluster demonstrates a high density of group members, a cluster leader simply invokes an adaptive localized broadcast within its cluster to disseminate multicast packets received from the source. Each group member in AAMRP can be in 3 states. It can be in a temporary mode wherein it is JOINING the session, it can be cluster LEADER, or it can simply be the MEMBER of a cluster leader.

\section{2 [2,6] Improved On Demand Multicast Routing Protocol (IODMRP)}

- In [6] an improved protocol named IODMRP which lessens shared bandwidth competition and network contention using decreasing ineffective data forwarding. It has a better end-to-end delay and delivery ratio than ODMRP with increasing senders. The establishing and updating of the forward structure in IODMRP is the same as ODMRP. The performance of IODMRP is better dramatically than ODMRP. It is exhibited through the smaller end to end delay and the higher packet delivery ratio with senders increasing. Redundant packets are less as compared to ODMRP.

- It is a more efficient multicast routing protocol.

- It chooses partial forwarding nodes to relay packets, the number of which is decided by probabilistic forwarding 
algorithm based on forwarder's density and the nodes are selected according to energy state.

- This protocol is implemented through simple modifications to existing ODMRP, but we reduce the redundant data transmissions and save energy significantly through decreasing the forwarding packets.

- It employs the algorithm of self-adapting probability, which means adjusting probability according to local density of forwarders. When the number of neighbor forwarding nodes is small the probability is $100 \%$ to guarantee high packet delivery ratio, on the contrary play down the probability to cut down partial contention and congestion, hence the transmission efficiency is heightened and the performance of the network is improved.

- The establishing and updating of the forward structure in IODMRP is the same as ODMRP.

\subsection{Security Model to be used}

\subsubsection{THE SB-TRUST MODEL:}

\section{CONSTRUCTION OF SB-PGP MODEL}

In this work, we apply the SB-model for issuing PGP type certificate. Let us consider a MANET, to be established, for instance, in a conference where people having mobile nodes communicate with one another having insecure wireless channel. We assume $\mathrm{N}$ mobile nodes, and $\mathrm{N}$ may be dynamically changing as mobile nodes join, leave, or fail over time. Among them, some of the nodes that joined in the beginning are considered as senior nodes and later joining nodes are considered junior nodes but the size of senior nodes group may increase dynamically and sequentially according to the size of network. Besides, $\mathrm{N}$ is constrained if there may be a large device population otherwise not.

Specifically, for the model construction, we make the following assumptions:

- Each node has a unique nonzero ID and a mechanism to discover available senior member nodes of the network.

- Communication with senior nodes is more reliable compared with junior nodes of the networks.

- Mobility is centralized by a maximum node moving speed Smax.

- Each senior node is equipped with some local detection mechanism to identify Misbehaving nodes among its surrounding nodes, e.g. those proposed in [1].

\section{SIMULATION ENVIRONMENT}

The aim of this simulation study is to compare the on-demand routing protocols i.e. Ant based Adaptive Multicast Routing Protocol (AAMRP) and Improved On Demand Multicast Routing Protocol(IODMRP) on the basis of various parameters as Packet delivery fraction, Throughput and End-to-end delay. The simulations have been performed using Network Simulator 2.0, software used to track simulations of wireless networks. This simulation is carried out within an Ad-hoc network taking the number of nodes is 50 and keeping the speed and pause time constant. Green line indicates AAMRP and Red line indicates IODMRP.
Table 1: SIMULATION ENVIRONMENT

Parameter

Value

\begin{tabular}{|l|l|}
\hline Simulation Time & $200 \mathrm{sec}$ \\
\hline No. of Nodes & $10,15,20,25,30,35,40,45,50$ \\
\hline Transmission Range & $250 \mathrm{~m}$ \\
\hline Traffic Type & CBR( Constant Bit Rate) \\
\hline Security Model & SBPGP \\
\hline Routing Protocols & IODMRP and AAMRP \\
\hline Observation Parameter & $\begin{array}{l}\text { Throughput, Packet Delivery } \\
\text { Fraction and End To End } \\
\text { Delay }\end{array}$ \\
\hline Mobility model & Random Waypoint \\
\hline MAC Protocol & IEEE 802.11 \\
\hline
\end{tabular}

\section{RESULT AND DISCUSSION}

To evaluate the performance of routing protocols, the following metrics are considered.

1) End-to-end delay: End-to-end delay indicates how long it took for a packet to travel from the source to the application layer of the destination. The variation of Average End-to-End Delay with varying the number of mobile nodes is shown in the Figure 1.

2) Packet Delivery Fraction: The fraction of packets sent by the application that are received by the receivers. The variation of Packet Delivery ratio with varying the number of mobile nodes is shown in the Figure 2.

3) Throughput: The total amount of data a receiver R actually receives from the sender divided by the time it takes for $R$ to get the last packet. The variation of Throughput with varying the number of mobile nodes is shown in the Figure 3. 


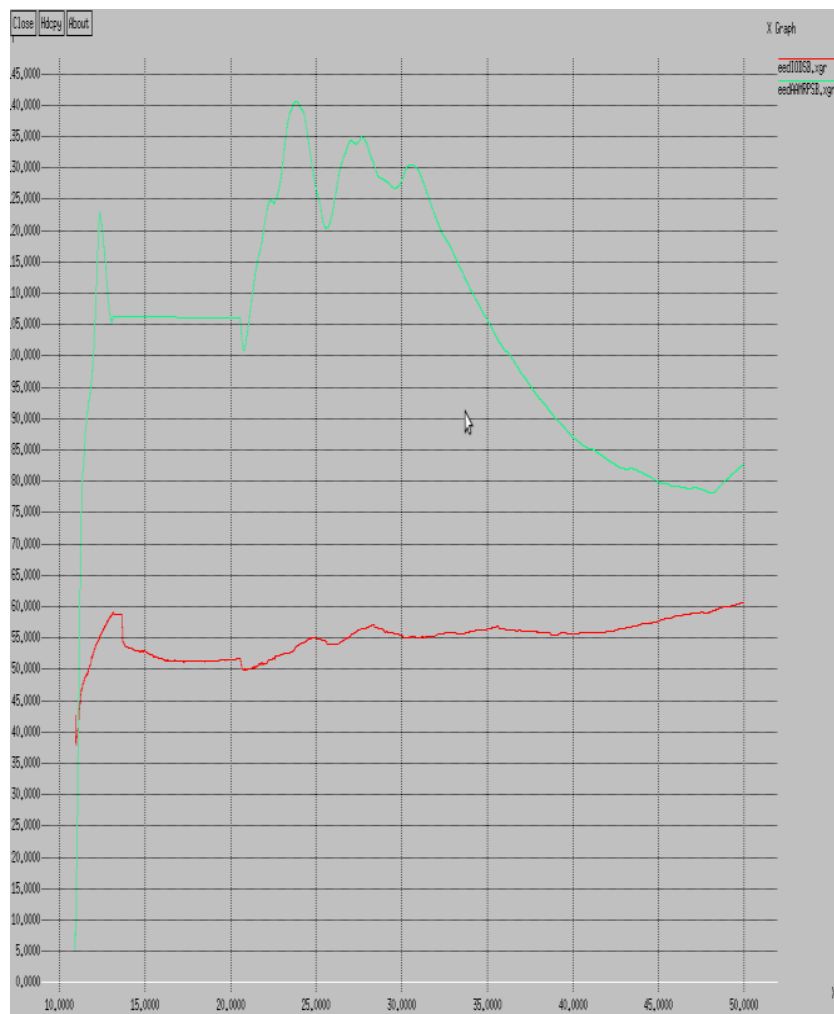

Figure 1: End-to-End Delay

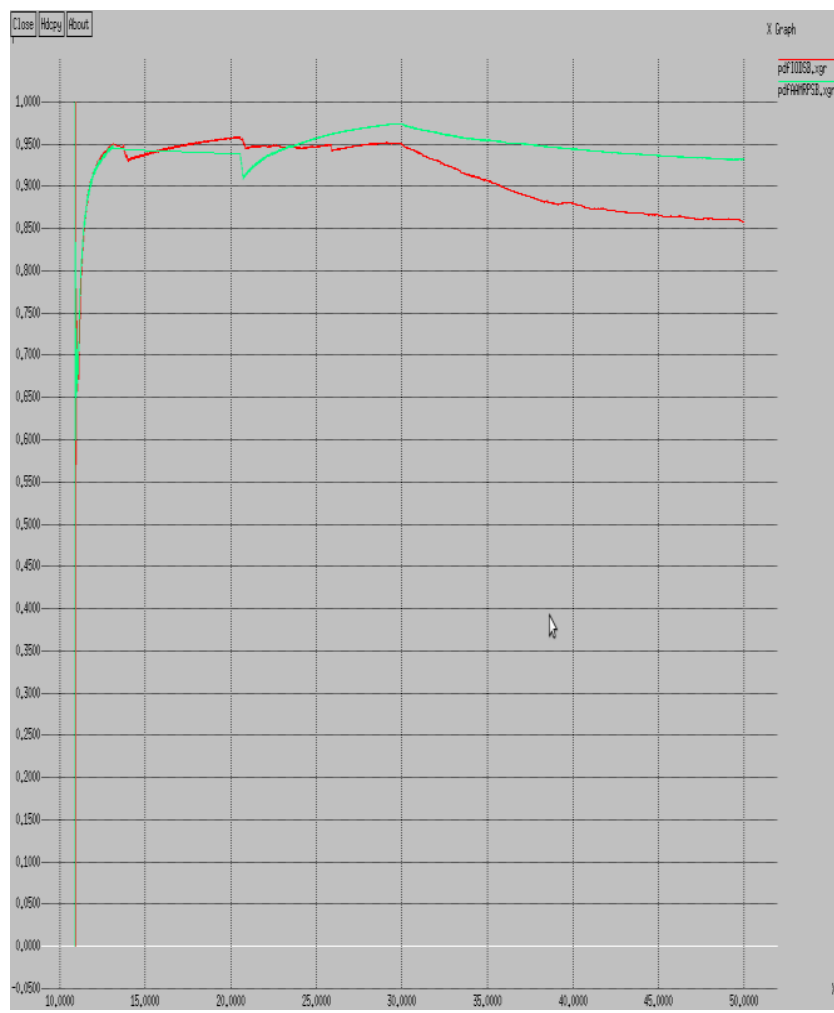

Figure 2: Packet Delivery Fraction

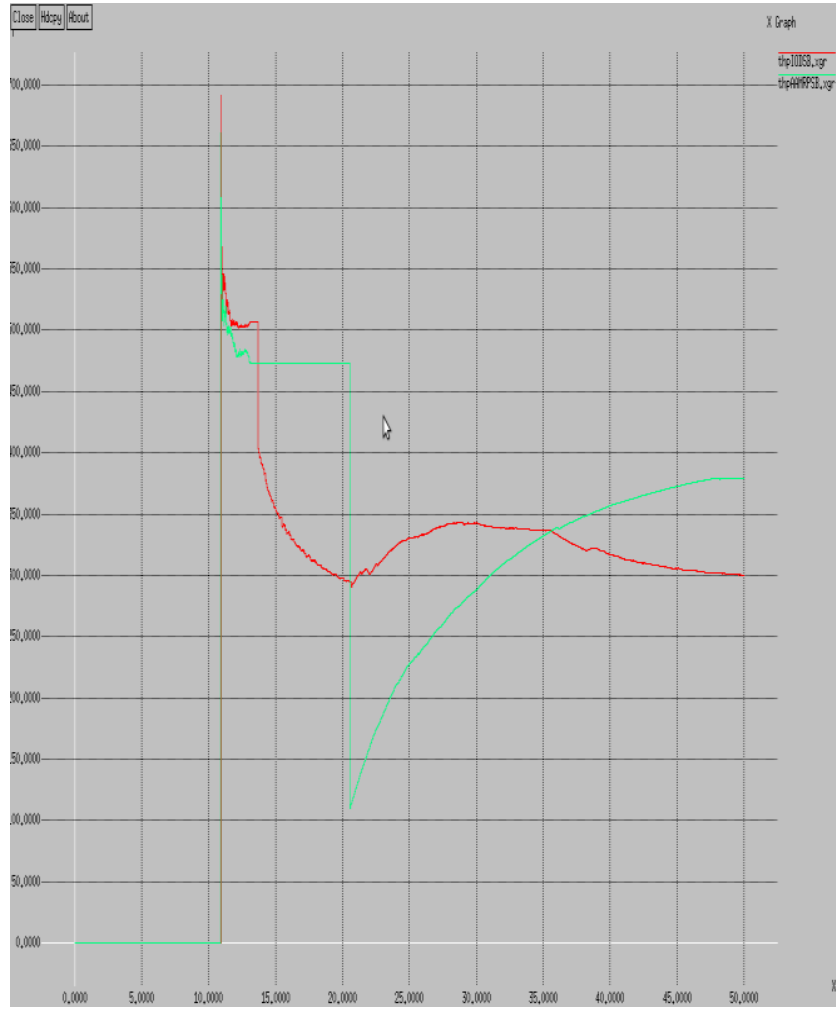

Figure 3: Throughput

\section{CONCLUSION}

In this current thesis work we have described the design of secure technique namely SBPGP with AAMRP and IODMRP protocols. It has been observed from the previous paper that the comparison of AAMRP and IODMRP was done without SBPGP security model. Current study is performed for comparison analysis for multicast routing protocols using SBPGP model for various QOS parameters. From the above graphs it has been concluded that AAMRP provides better QOS parameters with respect to IODMRP but in case of End-to-End Delay parameter IODMRP is somewhat better than AAMRP.

\section{FUTURE SCOPE}

In future analysis of AAMRP can be done with newly more effective multicast routing protocols as LBMRP (Location Based), DVMRP (Distance Vector) Multicast Routing Protocols for various different QOS parameters metrics be scrutinize.

\section{REFERENCES}

[1] Kimaya Sanzgiri, Daniel LaFlamme and Bridget Dahill, "Authenticated Routing for Ad hoc Networks" 'ICNP 2002.

[2] Moukhtar A. Ali and Ayman EL-SAYED ," A survey of Multicast routing protocols for Ad Hoc wireless networks", published in CMPE598 Graduate Seminars May 2,2006.

[3] S Gowrishankar ,T G Basavaraju and Subir Kumar Sarkar,"Effect of Random Mobility Models Patters in Mobile Ad Hoc Networks" published in IJCSNS International Journal Of Computer Science and Network Security,Vol. 7 No. 6,June 2007. 
[4] Khalid A. Farhan," On the scalability and Reliability of Network Sender Multicast Routing Protocol (NSMRP)" published in The fourth International Conference on Telecommunication, 2008.

[5] A. Sabari and K.Duraiswamy,"Ant Based Adaptive Multicast Routing Protocol (AAMRP) for Mobile Ad Hoc Networks",(IJCSIS) International Journal of Computer Science and Information Security, Vol. 6,No. 2,2009.

[6] Ying-xin $\mathrm{Hu}$ and Yu-feng Jia,'Improvement of wireless Multicast Routing with Energy-efficiency Based on
ODMRP", published in Computer network and multimedia technology in International Symposium,2009.

[7] Tanvir Kahlon and Sukesha Sharma " Comparison study on AAMRP and IODMRP in MANETS" published in (IJCSIS) International Journal of Computer Science and Information Security Vol.9,No.7,July2011.

[8] Shavinder, Sandeep Kang, "Comparative analysis of ODM and IODM Routing Protocols with PKC security model in MANETS”, IJECT Vol. 2,Issue 3, SEPT. 2011. 